University of Wollongong

Research Online

Faculty of Engineering and Information

Faculty of Engineering and Information

Sciences - Papers: Part A

Sciences

$1-1-2013$

Positron and electron collisions with nitrous oxide: measured and calculated cross sections

Luca Chiari

Flinders University,Australian National University

Antonio Zecca

University of Trento, Italy

Emanuele Trainotti

University of Trento, Italy

Gustavo Garcia

University of Wollongong

Francisco Blanco

Universidad Complutense, Avda. Complutense Sn, Madrid, Spain

See next page for additional authors

Follow this and additional works at: https://ro.uow.edu.au/eispapers

Part of the Engineering Commons, and the Science and Technology Studies Commons

Research Online is the open access institutional repository for the University of Wollongong. For further information contact the UOW Library: research-pubs@uow.edu.au 


\title{
Positron and electron collisions with nitrous oxide: measured and calculated cross sections
}

\author{
Abstract \\ We present cross-section results from an experimental and theoretical study on positron scattering from \\ nitrous oxide. Total cross sections (TCSs) have been measured at incident energies between 0.1 and 70 \\ $\mathrm{eV}$ with a linear-transmission-based positron spectrometer. Elastic differential and integral cross sections, \\ as well as inelastic integral cross sections and TCSs, have been computed with two different theoretical \\ approaches: the independent atom model with screening-corrected additivity rule (IAM-SCAR), at energies \\ in the 1- to 1000-eV range, and the Schwinger multichannel method at energies between 0.1 and $10 \mathrm{eV}$. \\ Note that the latter method specifically reports cross sections for the elastic channel. We find good \\ qualitative agreement between the theories and the TCS experiment at all common energies. That level of \\ accord is found to also become quantitative above the ionization energy of nitrous oxide. Electron-impact \\ TCSs calculated with the IAM-SCAR approach are also presented and compared to the existing results in \\ order to uncover any similarities or differences in the scattering processes between these two leptons \\ and nitrous oxide.

\section{Keywords} \\ oxide, calculated, electron, cross, sections, nitrous, collisions, measured, positron

\section{Disciplines} \\ Engineering | Science and Technology Studies

\section{Publication Details} \\ Chiari, L., Zecca, A., Trainotti, E., Garcia, G., Blanco, F., Bettega, M. H. F., Sanchez, S. d'A., Varella, M. T. do \\ N., Lima, M. A. P.. \& Brunger, M. J. (2013). Positron and electron collisions with nitrous oxide: measured \\ and calculated cross sections. Physical Review A: Atomic, Molecular and Optical Physics, 88 (2), \\ 022708-1-022708-10.
}

\section{Authors}

Luca Chiari, Antonio Zecca, Emanuele Trainotti, Gustavo Garcia, Francisco Blanco, Marcio H. F Bettega, Sergio d'A Sanchez, Marcio T. do N Varella, Marco A. P. Lima, and M J. Brunger 


\title{
Positron and electron collisions with nitrous oxide: Measured and calculated cross sections
}

\author{
Luca Chiari, ${ }^{1,2}$ Antonio Zecca, ${ }^{2}$ Emanuele Trainotti, ${ }^{2}$ Gustavo García, ${ }^{3,4}$ Francisco Blanco, ${ }^{5}$ Márcio H. F. Bettega, ${ }^{6}$ \\ Sergio d'A. Sanchez, ${ }^{6}$ Márcio T. do N. Varella, ${ }^{7}$ Marco A. P. Lima, ${ }^{8}$ and M. J. Brunger ${ }^{1,9}$ \\ ${ }^{1}$ ARC Centre for Antimatter-Matter Studies, School of Chemical and Physical Sciences, Flinders University, \\ GPO Box 2100, Adelaide, South Australia 5001, Australia \\ ${ }^{2}$ Department of Physics, University of Trento, Via Sommarive 14, 38123 Povo (Trento), Italy \\ ${ }^{3}$ Instituto de Física Fundamental, Consejo Superior de Investigationes Científicas (CSIC), Serrano 113-bis, 28006 Madrid, Spain \\ ${ }^{4}$ Centre for Medical Radiation Physics, University of Wollongong, Wollongong, New South Wales 2522, Australia \\ ${ }^{5}$ Departamento de Física Atómica, Molecular y Nuclear, Universidad Complutense de Madrid, 28040 Madrid, Spain \\ ${ }^{6}$ Departamento de Física, Universidade Federal do Paraná, Caixa Postal 19044, 81531-990 Curitiba, Paraná, Brazil \\ ${ }^{7}$ Instituto de Física, Universidade de São Paulo, Caixa Postal 66318, 05315-970 São Paulo, São Paulo, Brazil \\ ${ }^{8}$ Instituto de Física “Gleb Wataghin”, Universidade Estadual de Campinas, 13083-970 Campinas, São Paulo, Brazil \\ ${ }^{9}$ Institute of Mathematical Sciences, University of Malaya, 50603 Kuala Lumpur, Malaysia
}

(Received 12 July 2013; published 23 August 2013)

\begin{abstract}
We present cross-section results from an experimental and theoretical study on positron scattering from nitrous oxide. Total cross sections (TCSs) have been measured at incident energies between 0.1 and $70 \mathrm{eV}$ with a linear-transmission-based positron spectrometer. Elastic differential and integral cross sections, as well as inelastic integral cross sections and TCSs, have been computed with two different theoretical approaches: the independent atom model with screening-corrected additivity rule (IAM-SCAR), at energies in the 1- to 1000-eV range, and the Schwinger multichannel method at energies between 0.1 and $10 \mathrm{eV}$. Note that the latter method specifically reports cross sections for the elastic channel. We find good qualitative agreement between the theories and the TCS experiment at all common energies. That level of accord is found to also become quantitative above the ionization energy of nitrous oxide. Electron-impact TCSs calculated with the IAM-SCAR approach are also presented and compared to the existing results in order to uncover any similarities or differences in the scattering processes between these two leptons and nitrous oxide.
\end{abstract}

DOI: 10.1103/PhysRevA.88.022708

PACS number(s): $34.80 . \mathrm{Bm}, 34.80 . \mathrm{Gs}, 34.80 . \mathrm{Uv}$

\section{INTRODUCTION}

Nitrous oxide $\left(\mathrm{N}_{2} \mathrm{O}\right)$ is a well-known gas used for a wide range of industrial, engineering, medical, and recreational purposes. In the last quarter of a century, however, it has become of more environmental concern for both its large indirect reactivity with stratospheric ozone and its rapidly increasing atmospheric concentrations. It is now considered one of the major air pollutants in Earth's atmosphere, as it is the fourth-largest greenhouse gas, in terms of its anthropogenic emissions, and its global-warming potential over a 100 -year period is as large as 298 (compared to $\mathrm{CO}_{2}$ ) [1].

This study focuses on positron scattering from $\mathrm{N}_{2} \mathrm{O}$ and, hence, naturally extends the earlier work of our group on positron collisions with the major and trace gases of which the dry atmosphere of our planet is constituted, that is $\mathrm{N}_{2}$ and $\mathrm{CO}$ [2], $\mathrm{O}_{2}$ [3], $\mathrm{Ar}$ [4], $\mathrm{CO}_{2}$ [5], $\mathrm{CH}_{4}$ [6], $\mathrm{Kr}$ [7], $\mathrm{H}_{2}$ [8], and $\mathrm{Xe}$ [9]. Nitrous oxide is a linear triatomic molecule (Fig. 1) with a small permanent dipole moment $(\mu=0.161 \mathrm{D}[10])$ and a middle-sized dipole polarizability $\left(\alpha_{d}=20\right.$ a.u. [11]). Given the conclusions we drew in our previous studies on small-sized polar polyatomic molecules (see, e.g., [2,12]), these properties are nevertheless expected to affect the scattering process at low energies. The extent of this effect, in turn, is anticipated to become manifest in the shape and magnitude of the cross sections.

We note that earlier work on positron collisions with nitrous oxide is rather scarce and, hence, the present study aims at rectifying, at least in part, this lack of cross-section data. In fact, to the best of our knowledge there are only two available experimental investigations: The first reports total cross-section (TCS) results between 1 and $400 \mathrm{eV}$ [13], while the second gives quasielastic relative differential cross sections (DCSs) [14]. The number of theoretical studies is also very limited. In this respect we mention the paper of Baluja and Jain [15], who calculated the TCS using a local spherical complex optical potential approach. More recently Arretche et al. [16] reported elastic DCSs and integral cross sections (ICSs) as calculated using two different methods, namely the Schwinger multichannel (SMC) method and the method of continued fractions (MCF).

The situation with respect to electron scattering from $\mathrm{N}_{2} \mathrm{O}$ appears to be more detailed. A nearly exhaustive literature survey of the existing electron- $\mathrm{N}_{2} \mathrm{O}$ studies has been lately compiled by Vinodkumar and Barot [17] and, therefore, we do not list those works here again. For completeness, we note, however, that the aforementioned paper failed to cite the measurements of Kubo et al. [18] and Allan and Skalicky [19], the calculations of da Costa and Bettega [20] and Bettega et al. [21], as well as the recent data compilation from Anzai et al. [22].

Here we present new experimental TCSs for positron scattering from $\mathrm{N}_{2} \mathrm{O}$, measured with the positron spectrometer at the University of Trento, that extend the range of the available data down to $0.1 \mathrm{eV}$. We also carried out calculations of the elastic DCSs and ICSs, the inelastic ICSs, and the TCS for the positron- $\mathrm{N}_{2} \mathrm{O}$ system using the independent atom model with screening-corrected additivity rule (IAM-SCAR) and for the elastic DCSs and ICS using the SMC method. The 


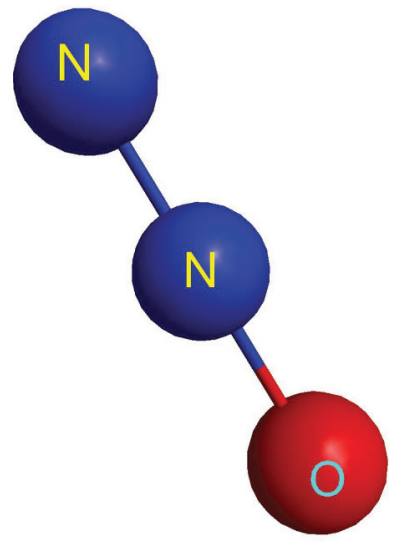

FIG. 1. (Color online) Geometric structure of the $\mathrm{N}_{2} \mathrm{O}$ molecule (generated using MCMOLPLT [64]).

combination of these two theories allows us to investigate this scattering system over a wide range of incident energies (from 0.1 to $1000 \mathrm{eV}$ ) and at different cross-section levels. Finally, we also present new TCS results for electron impact with $\mathrm{N}_{2} \mathrm{O}$, computed with our IAM-SCAR model, in order to compare the positron and electron scattering dynamics for this target.

The present paper is outlined as follows. In Sec. II we describe the experimental technique and analysis procedures employed in our measurements. In Sec. III we introduce our theoretical approaches to electron and positron scattering. Thereafter, in Sec. IV, we present and discuss our results. Finally, a summary of the main conclusions from this investigation is drawn.

\section{EXPERIMENTAL DETAILS}

The present positron TCS measurements were carried out with the positron facility at the University of Trento, which was developed by Zecca and collaborators and which has been described in detail many times (see, e.g., [2]). We, therefore, recall here only the key experimental techniques and data-analysis procedures. The experiment is based on a linear transmission technique, with a low-energy positron beam being produced by a radioactive ${ }^{22} \mathrm{Na}$ isotope (activity $\sim 1.5 \mathrm{mCi}$ ) in conjunction with a $1-\mu \mathrm{m}$-thick tungsten moderator [23]. A set of electrostatic optics and a weak magnetic field ( $B \approx 4-11 \mathrm{G}$ ) transport and focus the collimated beam into the scattering region where the positrons interact with the target molecules. Finally, a channel electron multiplier detects the positrons that exit the scattering cell.

The TCS of interest $(\sigma)$ is determined by measuring the attenuation of the beam intensity due to the interaction of the incident positrons with the target molecules and employing the Beer-Lambert law:

$$
I_{1}=I_{0} \exp \left(\frac{-\left(P_{1}-P_{0}\right) L \sigma}{k T}\right) .
$$

At any incident positron energy, the positron beam count rate $\left(I_{0}\right)$ and the pressure $\left(P_{0}\right)$ in the scattering region are measured when $\mathrm{N}_{2} \mathrm{O}$ is diverted into the vacuum chamber, i.e., away from the scattering cell. The same measurements of the positron count rate $\left(I_{1}\right)$ and the pressure $\left(P_{1}\right)$ are then repeated under the same conditions, but now with the $\mathrm{N}_{2} \mathrm{O}$ gas routed through the scattering cell. The temperature $(T)$ of the $\mathrm{N}_{2} \mathrm{O}$ gas in the scattering region is also measured using a platinum (PT100) resistance thermometer in thermal contact with the scattering chamber walls. The geometrical length of the scattering cell is $L=22.1 \pm 0.1 \mathrm{~mm}$, while $k$ is the Boltzmann constant.

Several experimental precautions are taken during the measurements. First, only a high-purity $\mathrm{N}_{2} \mathrm{O}$ source ( $>99.8 \%$ from BOC gases) was used throughout the present investigations. The target pressure in the scattering cell was set to such a value that the beam transmission (i.e., the ratio $I_{1} / I_{0}$ ) is greater than 0.7 in order to minimize multiple scattering events. In addition, it is standard practice in our laboratory to check for the validity of our techniques and procedures before every experiment on a new target is started. This is done by making preliminary validation measurements using targets for which the positron scattering TCSs are considered to be well known, such as the noble gases [4,7,9]. We also use molecular nitrogen [2] as a reference target for internal validation purposes.

The zero of the positron energy scale is calibrated through a retarding potential analysis (RPA) of the incident beam, without the target gas in the vacuum chamber, as described by Zecca and Brunger [24]. We estimate that the error on the energy scale is approximately $\pm 0.05 \mathrm{eV}$. The same RPA measurement also provides us with the energy distribution profile of the beam [24]. The energy width of the beam in this experiment was $\sim 0.25 \mathrm{eV}$ (FWHM), with an uncertainty of $\pm 0.05 \mathrm{eV}$ at most. Note that, given the finite energy resolution of the beam, our measured cross sections are actually the convolution of the "real" TCSs with the positron-beam energy distribution. However, this effect is expected to become significant only at very low energies (below $\sim 0.5 \mathrm{eV}$ ), where the incident positron energy is comparable to the energy width of the beam itself.

The collected data are also corrected for some instrumental effects before it is used in Eq. (1). The length of the scattering region $(L)$, for instance, is corrected to account for the path increase caused by the gyration of the positrons in the focussing axial magnetic field in the scattering region. This correction was accomplished through computations of the motion of charged particles in a magnetic field and assuming that the positrons have a distribution in the velocity component perpendicular to their direction of motion. For positron energies equal to and smaller than $25 \mathrm{eV}$ the magnetic field was set to $B \sim 11 \mathrm{G}$, which corresponds to an increase in $L$ of $5.5 \%$; for energies greater than $25 \mathrm{eV}$ the magnetic field was lowered to $B \sim 4 \mathrm{G}$ and so the correction was just $2 \%$. In addition, the gas pressure in the scattering cell is corrected for the thermal transpiration effect, as the readings were achieved with an MKS 627B capacitance manometer operating at $45^{\circ} \mathrm{C}$, whereas the gas temperature in the scattering cell was $\sim 22 \pm 2{ }^{\circ} \mathrm{C}$. This correction is done using the semiempirical method described by Takaishi and Sensui [25] and is smaller than $3.5 \%$ of the TCS magnitude. We note here that this model might actually somewhat overestimate the thermal transpiration correction for large molecules, such as 1,4-dioxane [26], compared to similar approaches available in the literature [27,28]. However, as $\mathrm{N}_{2} \mathrm{O}$ is a relatively small target (its hard-sphere diameter is $3.9 \AA$ [29]), we estimate that this effect would be very small $(<1 \%)$ in the pressure range 


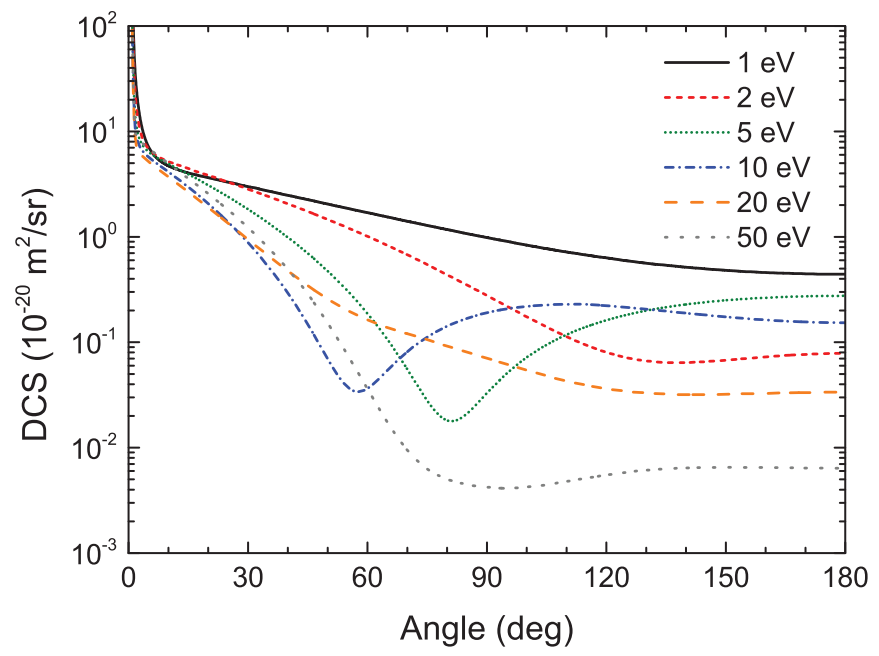

FIG. 2. (Color online) Present elastic DCSs for positron scattering from nitrous oxide, at selected energies between 1 and $50 \mathrm{eV}$, calculated with our IAM-SCAR approach averaged over rotational excitations.

and at the temperatures we worked at and in any case is well within our cited TCS errors. Therefore, we decided to continue to use the model of Takaishi and Sensui [25] here, as in our previous studies.

The forward angle scattering effect is known to play an important role in TCS measurements for positron scattering (see [30] for more details). Note that the present TCS measurements are uncorrected for this effect and, hence, represent a lower bound on the "real" value. The extent of the forward scattering effect depends on the angular discrimination of the apparatus and on the shape of the elastic DCSs for the target species of interest in this forward angular region [30]. The energy-dependent angular discrimination of the present apparatus is evaluated to vary between $17.5^{\circ}$ at $1 \mathrm{eV}$ and $2.4^{\circ}$ at $50 \mathrm{eV}$ positron energy (see Table II in Zecca et al. [2]). Note that these estimates compare quite well with those of the spectrometer at Wayne State University $\left(20^{\circ}\right.$ at $2 \mathrm{eV}$ and $8^{\circ}$ at $500 \mathrm{eV}$ [13]). The elastic DCSs for $\mathrm{N}_{2} \mathrm{O}$ are available from our IAM-SCAR computations averaged over the rotational excitations (Fig. 2) and our SMC calculations with Born dipole closure (Fig. 3) (see also Sec. III). Therefore, the present measured TCSs could, at least in principle, be corrected for the forward scattering effect using, for instance, the approach described by Hamada and Sueoka [31]. However, given that the agreement between the elastic DCSs calculated with our two methods is not uniformly good at all energies and that there are no independent experimental nor theoretical absolute elastic DCS results for positron- $\mathrm{N}_{2} \mathrm{O}$, we have, in general, not corrected our measured TCSs. Nevertheless, in order to provide an estimate of the extent of this effect, we have employed our theoretical elastic DCSs, computed with both approaches, to correct our measured TCSs at a few selected positron energies (see Figs. 4 and 5). Using the IAM-SCAR DCSs we find that our measured TCSs (Table I) would increase by $9.5 \%$ at $1 \mathrm{eV}$, to $4.9 \%$ at $10 \mathrm{eV}$, and $1.6 \%$ at $50 \mathrm{eV}$, whereas employing the SMC DCSs changes that correction from $81 \%$ at $0.1 \mathrm{eV}$ to $46 \%$ at $1 \mathrm{eV}$ and $12 \%$ at $10 \mathrm{eV}$. We note here that the large difference in the correction that we obtain at around

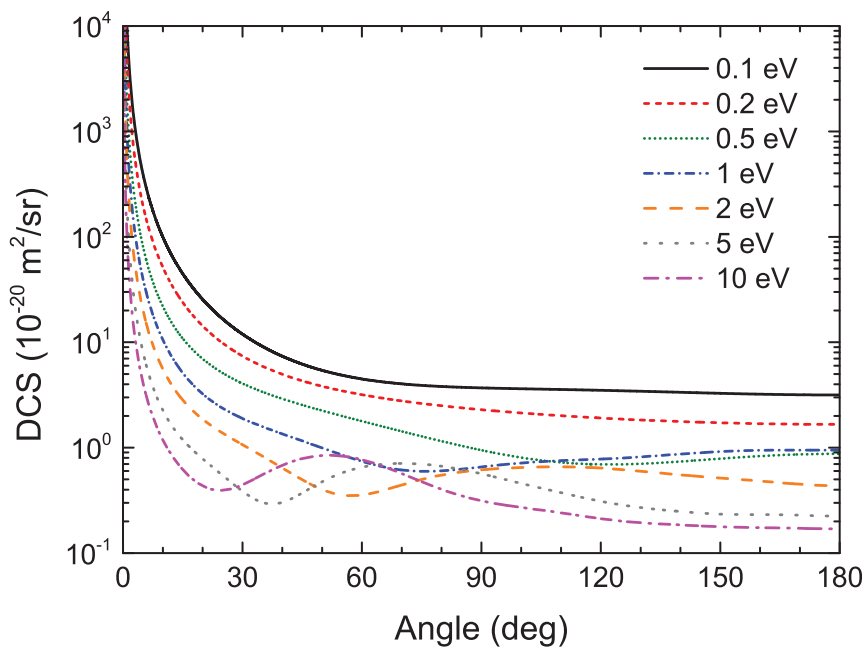

FIG. 3. (Color online) Present elastic DCSs for positron scattering from nitrous oxide, at selected energies from 0.1 to $10 \mathrm{eV}$, computed with our SMC + Born method.

$1 \mathrm{eV}$ with the two methods may be ascribed to the fact that the validity of our IAM-SCAR approach at those low-energies needs to be further investigated (see Sec. III).

We report the present experimental TCSs for energies between 0.1 and $70 \mathrm{eV}$. The statistical uncertainties are, on average, $2.3 \%$ in this energy range. The largest statistical errors amount to $5.5 \%$ and these are typically found at the lowest investigated energies only. The overall errors on the

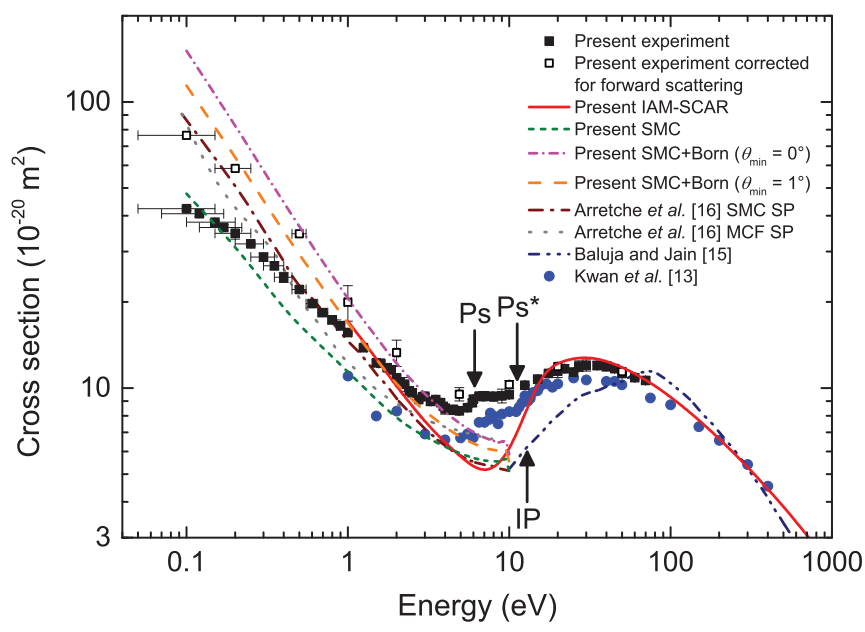

FIG. 4. (Color online) The present experimental TCS for positron scattering from nitrous oxide is compared to the TCS calculated with our IAM-SCAR method and the elastic ICSs computed with our SMC approach, without and with Born dipole closure. Shown are our SMC + Born elastic ICSs obtained by integrating the elastic DCSs from $\theta_{\min }=0^{\circ}$ and $\theta_{\min }=1^{\circ}$ (see text). Also plotted are the present experimental TCSs corrected for the forward scattering effect at selected energies (again, see text). The current results are also compared against the TCSs measured by Kwan et al. [13] and calculated by Baluja and Jain [15], as well as the theoretical elastic ICSs of Arretche et al. [16]. The threshold energies for Ps formation in its ground and first excited states, and the first ionization in $\mathrm{N}_{2} \mathrm{O}$, are indicated by the vertical black arrows denoted by "Ps", "Ps*", and "IP", respectively. 


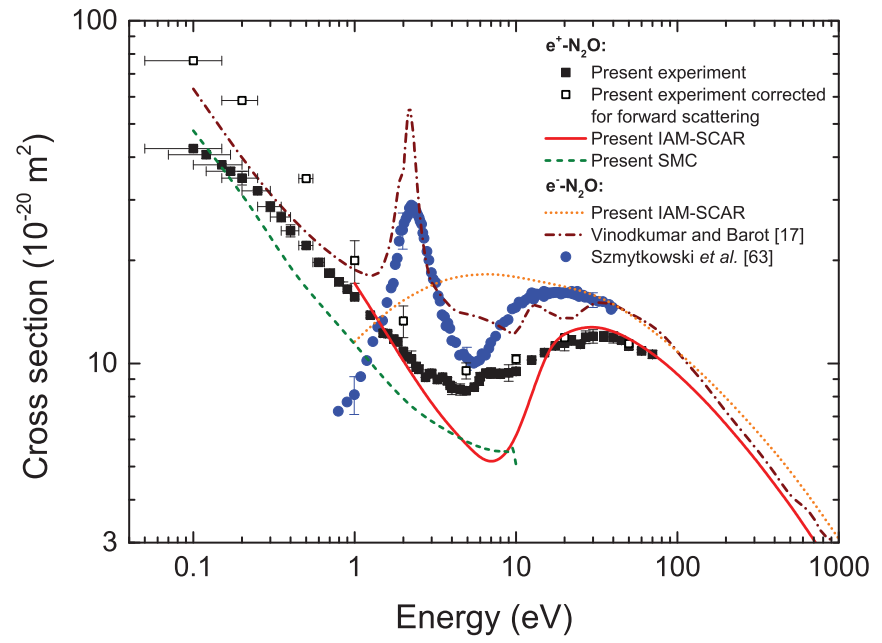

FIG. 5. (Color online) The present measured and calculated positron cross sections (see Fig. 4) are compared to the corresponding electron-impact results for nitrous oxide. Shown is the present electron TCS computed with our IAM-SCAR method, as well as the earlier electron TCSs measured by Szmytkowski et al. [63] and calculated by Vinodkumar and Barot [17].
TCSs are estimated to lie within the $\sim 4 \%-11 \%$ range. They originate from the statistical uncertainties, the uncertainty in the thermal transpiration correction $(<3 \%)$, the uncertainty in the length of the scattering region, as well as its correction for the effective positron path length $(<3 \%)$ and the uncertainty in the pressure and temperature readings $(<1 \%$ each $)$.

\section{THEORETICAL DETAILS}

\section{A. IAM-SCAR method}

\section{Atomic optical model for electron scattering}

The IAM-SCAR formalism has been widely employed in electron scattering calculations for a large number of different molecules (ranging from diatomics to biomolecules [32-36]), over a broad energy range, typically from 1 to $5000 \mathrm{eV}$. Hence, we only briefly summarize the method behind our calculations here. The first subjects of our computations are the individual atoms constituting the target molecule, i.e., $\mathrm{N}$ and $\mathrm{O}$ in this case. Our atomic optical model is based on a potential scattering approach, where the local complex potential $V(r)$ is

TABLE I. Present experimental TCSs for positron scattering from nitrous oxide. The errors represent the statistical uncertainties on the measurements.

\begin{tabular}{|c|c|c|c|c|c|}
\hline $\begin{array}{l}\text { Energy } \\
(\mathrm{eV})\end{array}$ & $\begin{array}{c}\text { TCS } \\
\left(10^{-20} \mathrm{~m}^{2}\right)\end{array}$ & $\begin{array}{c}\text { TCS error } \\
\left(10^{-20} \mathrm{~m}^{2}\right)\end{array}$ & $\begin{array}{c}\text { Energy } \\
(\mathrm{eV})\end{array}$ & $\begin{array}{c}\text { TCS } \\
\left(10^{-20} \mathrm{~m}^{2}\right)\end{array}$ & $\begin{array}{l}\text { TCS error } \\
\left(10^{-20} \mathrm{~m}^{2}\right)\end{array}$ \\
\hline 0.10 & 42.4 & 0.70 & 3.75 & 8.89 & 0.20 \\
\hline 0.12 & 40.7 & 1.00 & 3.90 & 8.63 & 0.10 \\
\hline 0.15 & 38.0 & 0.35 & 4.00 & 8.46 & 0.35 \\
\hline 0.17 & 36.4 & 0.86 & 4.50 & 8.40 & 0.32 \\
\hline 0.20 & 34.8 & 1.59 & 4.90 & 8.32 & 0.13 \\
\hline 0.25 & 31.9 & 0.36 & 5.50 & 8.53 & 0.09 \\
\hline 0.30 & 28.7 & 0.95 & 5.90 & 8.88 & 0.25 \\
\hline 0.35 & 26.8 & 0.88 & 6.00 & 9.14 & 0.25 \\
\hline 0.40 & 24.4 & 0.99 & 6.50 & 9.38 & 0.18 \\
\hline 0.50 & 22.1 & 0.45 & 6.90 & 9.33 & 0.11 \\
\hline 0.60 & 19.7 & 0.48 & 7.00 & 9.39 & 0.34 \\
\hline 0.70 & 18.4 & 0.14 & 7.90 & 9.36 & 0.18 \\
\hline 0.80 & 17.3 & 0.35 & 8.00 & 9.31 & 0.07 \\
\hline 0.90 & 16.5 & 0.27 & 9.00 & 9.39 & 0.52 \\
\hline 1.00 & 15.6 & 0.23 & 10.0 & 9.48 & 0.23 \\
\hline 1.25 & 13.8 & 0.26 & 12.5 & 10.2 & 0.03 \\
\hline 1.50 & 12.3 & 0.27 & 15.0 & 10.8 & 0.13 \\
\hline 1.60 & 12.2 & 0.21 & 17.5 & 11.3 & 0.10 \\
\hline 1.75 & 11.8 & 0.14 & 20.0 & 11.5 & 0.58 \\
\hline 1.85 & 11.6 & 0.33 & 22.0 & 11.7 & 0.38 \\
\hline 2.00 & 10.8 & 0.19 & 25.0 & 11.4 & 0.01 \\
\hline 2.10 & 10.5 & 0.06 & 27.0 & 11.9 & 0.51 \\
\hline 2.25 & 10.3 & 0.57 & 30.0 & 12.0 & 0.54 \\
\hline 2.40 & 9.78 & 0.31 & 35.0 & 12.0 & 0.40 \\
\hline 2.50 & 9.62 & 0.28 & 40.0 & 11.9 & 0.45 \\
\hline 2.75 & 9.11 & 0.00 & 45.0 & 11.7 & 0.23 \\
\hline 2.90 & 9.34 & 0.25 & 50.0 & 11.2 & 0.26 \\
\hline 3.00 & 9.36 & 0.19 & 60.0 & 10.9 & 0.12 \\
\hline 3.25 & 8.99 & 0.25 & 70.0 & 10.6 & 0.29 \\
\hline 3.50 & 9.12 & 0.08 & & & \\
\hline
\end{tabular}


given by

$$
V(r)=V_{\mathrm{s}}(r)+V_{\mathrm{ex}}(r)+V_{\mathrm{p}}(r)+i V_{\mathrm{a}}(r) .
$$

Here $V_{\mathrm{s}}(r)$ is the standard Hartree potential of the target, $V_{\text {ex }}(r)$ represents the exchange potential of Riley and Truhlar [37], $V_{\mathrm{p}}(r)$ is the dipole polarization potential employed by Zhang et al. [38], and $V_{\mathrm{a}}(r)$ is the imaginary absorption potential of Staszewska et al. [39]. Owing to this last term in Eq. (2), the optical model potential method yields a complex phase shift $\delta_{l}=\lambda_{l}+i \mu_{l}$. This enables us to calculate the (differential and integral) cross sections for elastic and inelastic (all excited electronic and ionized states combined together) scattering, as well as the grand TCS as the sum of those ICSs.

\section{Atomic optical model for positron scattering}

Our IAM-SCAR method for positron collisions with polyatomic molecules is based on an optical model potential approach similar to that defined above for electron impact and has been successfully employed in our recent positron scattering studies [3,36,40-42]. As the details of our approach have been described in those studies (see, in particular, [3]), here again we only recall its main points. In this case the local complex potential is given by

$$
V(r)=V_{\mathrm{s}}(r)+V_{\mathrm{p}}(r)+i V_{\mathrm{a}}(r) .
$$

As in Eq. (2), the real part describes the elastic scattering process and consists of the electrostatic interaction term, $V_{\mathrm{s}}(r)$, and the polarization term, $V_{\mathrm{p}}(r)$. The imaginary part, $V_{\mathrm{a}}(r)$, accounts for inelastic processes (including now positronium formation), which are considered as absorptions from the incident positron beam.

The static interaction was calculated from the charge density derived from Hartree-Fock atomic wave functions, using a similar procedure to that of Reid and Wadehra [43-45]. The choice of the polarization potential is particularly important in positron scattering calculations, as that is the only attractive contribution to the positron-atom interaction (except for virtual positronium formation). Here we use a new dipole plus quadrupole polarization potential, based on that developed by McEachran et al. [46] for $\mathrm{Ne}$, but scaled by a constant in order to give the known dipole and quadrupole $\left(\alpha_{\mathrm{q}}\right)$ polarizabilities of the $\mathrm{N}\left(\alpha_{\mathrm{d}}=7.42 \mathrm{au}, \alpha_{\mathrm{q}}=25.66 \mathrm{au}\right)$ and $\mathrm{O}$ $\left(\alpha_{\mathrm{d}}=5.41 \mathrm{au}, \alpha_{\mathrm{q}}=16.90 \mathrm{au}\right)$ atoms $[47,48]$. These scaling parameters are 2.276 and 2.632 for the dipole and quadrupole polarization potential of $\mathrm{O}$ and 3.122 and 3.996 for those of $\mathrm{N}$, respectively.

The definition of the energy for the absorption threshold $(\Delta)$ is also critical owing to the unique positronium (Ps) formation channel in positron scattering. While for electron scattering $\Delta$ would correspond to the excitation energy of the first electronic state of the atom in question, for positron collisions Ps formation is a dominant inelastic scattering channel that generally becomes open at a lower energy than that of the first excited electronic level. As Ps formation cannot be explained in terms of binary collisions [49], it cannot be explicitly introduced into the absorption potential as an independent inelastic process. Here we use an energy-dependent parameter for the absorption threshold $[\Delta(E)]$ of the form

$$
\Delta(E)=\Delta-\left(\Delta-\Delta_{\mathrm{p}}\right) e^{-\left(\frac{E-\Delta_{\mathrm{p}}}{E_{\mathrm{m}}}\right)} .
$$

In Eq. (4), $\Delta$ is the lowest excitation energy of the atom, $\Delta_{p}$ is the Ps formation threshold energy, and $E_{\mathrm{m}}$ is a characteristic energy at which the absorption potential, without Ps formation, gives the maximum inelastic ICS (here $E_{\mathrm{m}}=30 \mathrm{eV}$ ). Note that $\Delta(E)=\Delta_{\mathrm{p}}$ for energies around the Ps formation energy and $\Delta(E)=\Delta$ at higher energies. Further note that our results are not very sensitive to the choice of $E_{\mathrm{m}}$.

\section{Screening-corrected additivity rule}

In order to calculate the cross sections for electron and positron scattering from the $\mathrm{N}_{2} \mathrm{O}$ molecule, we then applied the additivity rule (AR) technique to our IAM results for each constituent atom. In this approach, the molecular scattering amplitude is derived from the sum of all the relevant atomic amplitudes, including the phase coefficients, leading this way to the DCSs for the molecule in question. ICSs can then be determined by integrating those DCSs, with the sum of the elastic ICS and absorption ICS (for all inelastic processes except rotations and vibrations) then giving the TCS. However, the AR does not take into account the molecular structure, so that it is really only applicable when the incident particles are so fast that they effectively "see" the target molecule as a sum of the individual atoms (typically above $\sim 100 \mathrm{eV}$ ). In order to partially overcome this limitation, Blanco and García [35,50] introduced the SCAR method. This formalism accounts for the geometry of the molecule (atomic positions and bond lengths) by using some screening coefficients. With this correction the range of validity might be extended to impact energies of $\sim 30 \mathrm{eV}$ for electron scattering and to perhaps somewhat higher energies for positron scattering. Further work is needed in the case of positrons to specify what the lower bound on the validity of the SCAR method actually is.

\section{Rotational excitations}

The IAM-SCAR approach described above does not account for vibrational and rotational excitations. However, for polar molecules such as $\mathrm{N}_{2} \mathrm{O}$, additional dipole-induced excitation cross sections can be calculated following the procedure suggested by Jain [51]. In that approach, rotational excitation DCSs and ICSs for a free electric dipole are calculated in the framework of the first Born approximation (FBA). These results can then be incorporated into our IAMSCAR calculation in an incoherent way, just by adding up the cross sections as independent channels. Although rotational excitation energies are, in general, very small (typically a few $\mathrm{meV}$ ) in comparison with the present incident energies, the incident energies should be higher than about a few $\mathrm{eV}$ in order to validate the Born approximation. Under these circumstances, rotational excitation cross sections $J \rightarrow J^{\prime}$ were calculated by weighting the population for the $J$ th rotational quantum number at $300 \mathrm{~K}$ and estimating the average excitation energy from the corresponding rotational constants.

\section{B. SMC method}

To compute the elastic scattering cross sections we also employed the SMC method as implemented for collisions of positrons with molecules. The SMC method has been 
described in detail in several publications [52-55] and so here we discuss only the relevant points to the present calculations.

The SMC method is a variational method to the scattering amplitude. The final expression to the scattering amplitude in the molecular reference frame is

$$
f\left(\vec{k}_{f}, \vec{k}_{i}\right)=-\frac{1}{2 \pi} \sum_{m, n}\left\langle S_{\vec{k}_{f}}|V| \chi_{m}\right\rangle\left(d^{-1}\right)_{m n}\left\langle\chi_{n}|V| S_{\vec{k}_{i}}\right\rangle
$$

where

$$
d_{m n}=\left\langle\chi_{m}\left|A^{(+)}\right| \chi_{n}\right\rangle
$$

and

$$
A^{(+)}=Q \hat{H} Q+P V P-V G_{P}^{(+)} V .
$$

In the above equations, $\left|S_{\vec{k}_{i, f}}\right\rangle$ is a solution of the unperturbed Hamiltonian $H_{0}$ (kinetic energy of the incoming positron plus the target Hamiltonian, $T_{N+1}+H_{N}$ ) and is a product of a target state and a plane wave $\left(|\vec{k}\rangle \otimes\left|\Phi_{i}\right\rangle\right) ; V$ is the interaction potential between the incident positron and the electrons and nuclei of the target; $\left\{\left|\chi_{m}\right\rangle\right\}$ is a basis set of $(N+1)$-particle states [configuration state functions (CSFs)] used to expand the trial scattering wave function; $\hat{H}=E-H$ is the collision energy minus the full Hamiltonian of the system, with $H=H_{0}+V ; P$ is a projection operator onto the open-channel space defined by the target eigenfunctions; and $G_{P}^{(+)}$is the free-particle Green's function projected on to the $P$ space. $Q=(\mathbb{1}-P)$ is the projector onto the closed electronic channels of the target, which are used in the description of polarization effects.

For calculations in the static approximation (where the distortion of the electronic cloud of the target due to the incoming positron is completely neglected) the direct space is constructed considering CSFs of the form

$$
\left|\chi_{i}\right\rangle=\left|\Phi_{1}\right\rangle \otimes\left|\varphi_{i}\right\rangle
$$

where $\left|\Phi_{1}\right\rangle$ is an $N$-electron Slater determinant of the target ground state obtained at the Hartree-Fock level and $\left|\varphi_{i}\right\rangle$ is a single-particle function which represents the incoming positron. The set composed by these single-particle functions are used as the scattering orbitals.

Polarization effects are taken into account by enlarging the direct space with CSFs from the closed-channel space of the form

$$
\left|\chi_{i j}\right\rangle=\left|\Phi_{i}\right\rangle \otimes\left|\varphi_{j}\right\rangle,
$$

where $\left|\Phi_{i}\right\rangle$ is an $N$-particle Slater determinant obtained by performing single (virtual) excitations from the occupied molecular (hole) orbitals to a set of unoccupied molecular (particle) orbitals. The $\left|\varphi_{j}\right\rangle$ is again a single-particle function used as a scattering orbital. The choice of the particle and scattering orbitals is discussed below.

Our calculations were performed in the static-polarization approximation in the $C_{2 v}$ symmetry group. We employed the ground-state equilibrium geometry of the molecule as given in Ref. [10]. We used the $\mathrm{TZV}+\mathrm{G}(3 d)$ basis set for nitrogen and oxygen. In particular, since the $\mathrm{N}_{2} \mathrm{O}$ molecule is linear and this $\mathrm{TZV}+\mathrm{G}(3 d)$ basis set contains only $s^{-}, p-$, and $d$-type functions, it describes only partial waves with azimuthal quantum numbers up to $m=2$. In order to account for the contribution of the $m=3$ partial wave, we followed the procedure used by Bettega et al. [21] for electron collisions with $\mathrm{N}_{2} \mathrm{O}$. We considered 24 extra chargeless centers located at the vertices of four hexagons with sides equal to $1.1 \AA$, that were placed in planes perpendicular to the internuclear axis and halfway between the atoms and half a bond length beyond the nuclei. At each of these centers we included one $s$-type function with exponent 0.144 and one $p$-type function with exponent 0.2 .

As noted above, to take polarization effects into account we considered single excitations from the hole orbitals to a set of particle orbitals. To represent these orbitals we employed modified virtual orbitals (MVOs) [56], obtained from a cationic Fock operator with charge +6 . We considered all the valence occupied orbitals as hole orbitals and excitations to the lowest 54 MVOs, which were also used, along with the occupied orbitals, as scattering orbitals. We obtained 7684 CSFs for the $A_{1}$ symmetry, 7069 CSFs for the $B_{1}$ symmetry, $7061 \mathrm{CSF}$ for the $B_{2}$ symmetry, and $6461 \mathrm{CSF}$ for the $A_{2}$ symmetry.

The calculated permanent electric dipole moment of the target was $0.619 \mathrm{D}$, which is larger than the experimental value of 0.161 D [10]. The SMC method uses only square integrable $\left(L^{2}\right)$ functions in the expansion of the scattering wave function. Those $L^{2}$ functions cannot exactly describe the long-range dipole interaction and, in this case, the extra centers also help to increase the range of the interaction potential in the SMC calculations. We used the standard Born closure scheme to better describe the dipole potential [57], where we computed the scattering amplitude for a point dipole potential in the FBA. We then expanded the scattering amplitude obtained with the SMC method in partial waves up to a given $\ell_{\text {SMC }}$. The FBA amplitude of the dipole potential is also expanded in partial waves. The Born closure is achieved by keeping the low partial waves (up to $\ell_{\text {SMC }}$ ), obtained from the SMC amplitude, and summing the high partial waves (from $\ell_{\mathrm{SMC}}+1$ to $\infty$ ) obtained from the FBA dipole amplitude. The choice of $\ell_{\text {SMC }}$ was made in order to minimize the difference between the DCSs obtained using the Born closure and the SMC method at high scattering angles. In the present calculations, we chose $\ell_{\mathrm{SMC}}=1$ from 0.1 to $0.5 \mathrm{eV}, \ell_{\mathrm{SMC}}=2$ from 1.0 to $1.5 \mathrm{eV}$, $\ell_{\mathrm{SMC}}=4$ from 2.0 to $2.5 \mathrm{eV}, \ell_{\mathrm{SMC}}=5$ from 3.0 to $3.55 \mathrm{eV}$, and $\ell_{\text {SMC }}=6$ from 4.0 to $10 \mathrm{eV}$. To obtain the ICS we then integrated the DCSs from $\theta_{\min }=0^{\circ}$ or $1^{\circ}$ up to $180^{\circ}$ [57]. We note here that in the calculation of our DCSs using the Born closure method the rotational constant was $5.5 \times 10^{-5} \mathrm{eV}$ (twofold degenerate).

\section{RESULTS AND DISCUSSION}

In Table I and Figs. 4 and 5 we present our measured TCSs for positron scattering from $\mathrm{N}_{2} \mathrm{O}$. Also shown in Figs. 4 and 5 are the experimental TCSs corrected for the forward angle scattering effect at selected incident energies. Note that the error bars on the corrected TCSs shown between 1 and $10 \mathrm{eV}$ reflect the difference in the elastic DCSs, as computed with the two present theoretical approaches (compare Figs. 2 and 3), that were used to calculate the correction at each energy (see Sec. II). We see in Fig. 4 that the TCS magnitude markedly decreases from the lowest investigated energy up 
TABLE II. Present positron- $\mathrm{N}_{2} \mathrm{O}$ TCSs $\left(\sigma_{\mathrm{T}}\right)$ and ICSs $\left(\right.$ all in $10^{-20} \mathrm{~m}^{2}$ ) for elastic scattering $\left(\sigma_{\mathrm{E}}\right)$, the inelastic processes (sum of electronic excitations, positronium formation, and direct ionization) $\left(\sigma_{\mathrm{I}}\right)$, and the rotational excitations $\left(\sigma_{\mathrm{R}}\right)$, as calculated with our IAM-SCAR approach.

\begin{tabular}{lccccccccc}
\hline \hline Energy $(\mathrm{eV})$ & $\sigma_{\mathrm{E}}$ & $\sigma_{\mathrm{I}}$ & $\sigma_{\mathrm{R}}$ & $\sigma_{\mathrm{T}}$ & Energy $(\mathrm{eV})$ & $\sigma_{\mathrm{E}}$ & $\sigma_{\mathrm{I}}$ & $\sigma_{\mathrm{R}}$ \\
\hline 1 & 16.1 & 0 & 1.08 & 17.2 & 40 & 3.39 & 9.02 & 0.03 \\
1.5 & 11.9 & 0 & 0.76 & 12.7 & 50 & 3.28 & 8.54 & 0.03 \\
2 & 9.55 & 0 & 0.59 & 10.1 & 70 & 3.00 & 7.70 & 0.03 \\
3 & 7.20 & 0 & 0.41 & 7.61 & 100 & 2.57 & 6.75 & 0.03 & 9.4 \\
4 & 6.13 & 0 & 0.32 & 6.45 & 150 & 2.06 & 5.68 & 0 \\
5 & 5.49 & 0 & 0.26 & 5.75 & 200 & 1.73 & 4.93 & 0 \\
7 & 4.73 & 0 & 0.19 & 4.92 & 300 & 1.34 & 3.98 & 0 \\
10 & 4.03 & 1.56 & 0.14 & 5.73 & 400 & 1.10 & 3.33 & 0 \\
15 & 3.19 & 7.56 & 0.08 & 10.8 & 500 & 0.95 & 2.91 & 0.35 \\
20 & 3.25 & 9.10 & 0.08 & 12.4 & 700 & 0.75 & 2.31 & 0 \\
30 & 3.42 & 9.44 & 0.06 & 12.9 & 1000 & 0.58 & 1.79 & 0 \\
\hline \hline
\end{tabular}

to nearly the Ps formation threshold energy in $\mathrm{N}_{2} \mathrm{O}$, that is $6.1 \mathrm{eV}$. As we anticipated in Sec. I, this behavior in the low-energy TCS was not unexpected. In fact, in our earlier studies on small-sized polar polyatomic molecules, such as in Refs. [2,12], we observed that the permanent dipole moment and the dipole polarizability of the target play a critical role in the scattering dynamics at those low energies. As the Ps formation and, subsequently, the first ionization (threshold energy $12.9 \mathrm{eV}$ [58]) channels become open, the TCS rises in magnitude and reaches a maximum at about $30-40 \mathrm{eV}$ before it starts decreasing in magnitude again. There appears to be also a statistically significant increase in the present TCS data at around $11 \mathrm{eV}$. Given that the energy of the first excited state of Ps is $5.1 \mathrm{eV}$ above that for the ground state, this rise might be associated to Ps formation in its first excited state. However, at around the threshold energies for the ground and first excited states of Ps the scattering channels corresponding to some of the electronic excited states of $\mathrm{N}_{2} \mathrm{O}$ [59-61] also become open, such as the $\mathrm{C}^{1} \Pi$ and $\mathrm{D}^{1} \Sigma^{+}$states with thresholds at about 8.5 and $9.6 \mathrm{eV}$ [62]. Therefore, they might also somewhat contribute to the steps observed in our data at around 6.1 and $11 \mathrm{eV}$.

Also shown in Fig. 4 are the results of the previous experimental [13] and theoretical $[15,16]$ work on $\mathrm{N}_{2} \mathrm{O}$. Good qualitative agreement is found between the present measured TCS and the data of Kwan et al. [13], although our results appear larger in magnitude than theirs. This disagreement with the data of Kwan et al. [13] can easily be addressed in terms of the inferior angular discrimination of the apparatus used in their measurements, compared to that of the Trento spectrometer (see Sec. II). In other words, the TCS of Kwan et al. [13] would need a larger correction, with respect to the Trento data, to account for the forward angle scattering effect. We would also not expect very good agreement with the results of Arretche et al. [16] above the Ps formation threshold, as those calculations are at the elastic ICS level only. This is precisely what we observe in Fig. 4. However, below that threshold we still observe some discrepancy between their calculations and the present experimental TCS, which cannot be merely explained in terms of contributions from vibrational excitations. The discrepancies between the present measured TCS and those elastic ICSs from Arretche et al. [16], as well as with the TCS results of Baluja and Jain [15], most likely indicates that some improvements in those theoretical approaches might be warranted.

In order to address, at least in part, the above limitations in the existing positron- $\mathrm{N}_{2} \mathrm{O}$ models, we carried out new calculations for this scattering system (see Fig. 4). These new calculations were obtained with our IAM-SCAR approach and our SMC method without and with Born closure (the latter denoted as "SMC + Born" hereafter). Table II therefore

TABLE III. Present elastic ICSs $\left(10^{-20} \mathrm{~m}^{2}\right)$ for positron scattering from nitrous oxide as computed with our SMC method without and with Born dipole closure (the latter obtained by integrating the elastic DCSs from $\theta_{\min }=0^{\circ}$ ).

\begin{tabular}{|c|c|c|c|c|c|}
\hline Energy (eV) & SMC & $\mathrm{SMC}+$ Born & Energy $(\mathrm{eV})$ & SMC & $\mathrm{SMC}+$ Born \\
\hline 0.1 & 47.8 & 151 & 4.5 & 6.05 & 7.81 \\
\hline 0.2 & 31.4 & 82.8 & 5 & 5.90 & 7.48 \\
\hline 0.3 & 23.4 & 57.5 & 5.5 & 5.78 & 7.22 \\
\hline 0.4 & 19.1 & 44.5 & 6 & 5.70 & 7.02 \\
\hline 0.5 & 16.5 & 36.7 & 6.5 & 5.63 & 6.85 \\
\hline 1 & 11.4 & 20.4 & 7 & 5.58 & 6.71 \\
\hline 1.5 & 9.09 & 15.1 & 7.5 & 5.55 & 6.61 \\
\hline 2 & 7.86 & 12.1 & 8 & 5.54 & 6.53 \\
\hline 2.5 & 7.19 & 10.6 & 8.5 & 5.56 & 6.49 \\
\hline 3 & 6.77 & 9.51 & 9 & 5.46 & 6.34 \\
\hline 3.5 & 6.47 & 8.82 & 9.5 & 5.88 & 6.71 \\
\hline 4 & 6.24 & 8.22 & 10 & 5.06 & 5.85 \\
\hline
\end{tabular}


TABLE IV. Present electron- $\mathrm{N}_{2} \mathrm{O}$ TCSs calculated with our IAMSCAR approach.

\begin{tabular}{lccc}
\hline \hline $\begin{array}{l}\text { Energy } \\
(\mathrm{eV})\end{array}$ & $\begin{array}{c}\text { TCS } \\
\left(10^{-20} \mathrm{~m}^{2}\right)\end{array}$ & $\begin{array}{c}\text { Energy } \\
(\mathrm{eV})\end{array}$ & $\begin{array}{c}\text { TCS } \\
\left(10^{-20} \mathrm{~m}^{2}\right)\end{array}$ \\
\hline 1 & 11.6 & 40 & 14.9 \\
1.5 & 13.9 & 50 & 14.0 \\
2 & 15.3 & 70 & 12.5 \\
3 & 17.0 & 100 & 10.9 \\
4 & 17.8 & 150 & 9.10 \\
5 & 18.1 & 200 & 7.95 \\
7 & 18.3 & 300 & 6.47 \\
10 & 17.9 & 400 & 5.52 \\
15 & 17.2 & 500 & 4.86 \\
20 & 16.8 & 700 & 3.93 \\
30 & 15.9 & 1000 & 3.08 \\
\hline \hline
\end{tabular}

reports the present IAM-SCAR results for the elastic and inelastic (sum over electronic excitations, Ps formation, and direct ionization) ICSs, the ICS for rotational excitation and the TCS. In Table III we give the elastic ICS values computed with our SMC and SMC+Born formalism. A common feature of all our calculations is the monotonic decrease in the cross section from the lowest energy up to $10 \mathrm{eV}$ for the SMC elastic ICS and up to the opening of the Ps formation channel in the IAM-SCAR TCS. At higher energies, the behavior of the IAM-SCAR TCS resembles quite well that of our experimental data. In fact, above $\sim 15 \mathrm{eV}$ that computation is in good quantitative agreement with our measured TCS, to within the overall uncertainties on the experiment. However, below that energy the IAM-SCAR calculation diverges from the experiment as it is consistently lower in magnitude, except at the lowest computed energies. This observation is consistent with our caveat in Sec. III that, a priori, our IAM-SCAR approach is not expected to be valid at such low energies. Nonetheless, at energies above $100 \mathrm{eV}$ we see excellent agreement between the IAM-SCAR calculation and the data of Kwan et al. [13]. At these higher energies, the forward angle scattering correction to the measured data would be very small, so that this observed agreement is robust here. We also see in Fig. 4 the relevance of the Born closure method in enhancing the magnitude of the elastic ICSs computed with our SMC method, in particular at the very-low energies. To assess in more detail the extent of the effect of the Born closure in our calculation, we also calculated our elastic ICS by integrating the elastic DCSs from $\theta_{\text {min }}=1^{\circ}$ instead of $\theta_{\min }=0^{\circ}$ (Fig. 4). As expected, this latter calculation is found to lie between the SMC and SMC + Born elastic ICSs. Given that the experimental value of the permanent dipole moment for $\mathrm{N}_{2} \mathrm{O}$ is very small and as the calculated value with our $\mathrm{SMC}$ approach is much larger than the experimental value, we believe that the results obtained with the SMC method without Born closure are possibly most representative of the experimental conditions. The level of accord between our measured TCS and the SMC elastic ICSs below the threshold energy of the first inelastic process is mostly qualitative only, although it seems that the SMC+Born calculation might agree with our corrected experimental data between about 0.5 and $2 \mathrm{eV}$.
In order to provide a better insight into the low-energy lepton scattering process from $\mathrm{N}_{2} \mathrm{O}$, we compare in Fig. 5 the present experimental and theoretical positron-impact results to a selection of the electron-impact TCSs available in the literature. Specifically, in that figure we show the electron TCS computed by Vinodkumar and Barot [17] with their $R$-matrix formalism and the TCS measured by Szmytkowski et al. [63]. To aid this positron-electron comparison we also carried out additional IAM-SCAR calculations for electron scattering from $\mathrm{N}_{2} \mathrm{O}$ (also shown in Fig. 5 and listed in Table IV), as outlined in Sec. III. First we note that the present computed electron TCS compares quite well to the existing electron results shown in Fig. 5 from the highest investigated energy down to about $20 \mathrm{eV}$. Below this energy, however, our calculation diverges from those previous cross sections and, in particular, does not reproduce the broad resonance centered at $\sim 2.3 \mathrm{eV}$. This is no surprise as, by its inherent construct, the IAM-SCAR approach cannot describe the temporary capture by the target of the incident electron, which leads to resonant phenomena. In addition, our IAM-SCAR electron computation seems to suggest that the TCS would decrease in magnitude below $1 \mathrm{eV}$ as one goes to further lower energies, which is in contrast to the most recent calculation by Vinodkumar and Barot [17] and contrary to what one would expect given the important role of the dipole moment and dipole polarizability in the scattering dynamics. It is interesting to note that the measured and calculated positron and electron-impact cross sections of Ref. [17], in Fig. 5, display the same energy dependence at least below $\sim 1 \mathrm{eV}$, which suggests that the behavior of the TCS at these very low energies might be quite insensitive to the charge of the projectile and rather be largely driven by the dipole interaction. Although one would expect the electron TCS to be larger in magnitude than the corresponding positron TCS in that energy range, due to the exchange interaction that is available in the electron scattering case, the existing data nonetheless show a mixed picture. While the electron TCSs of Vinodkumar and Barot [17] do appear larger in magnitude than the present positron cross sections, this is true only if one does not account for the forward angle scattering correction to the positron TCS data. Above $\sim 2 \mathrm{eV}$ the positron cross sections seem to lie consistently below the electron cross sections. The presence of a $\pi^{*}$ resonance at around $2 \mathrm{eV}$ and a $\sigma$ resonance at around $8 \mathrm{eV}$ in the electron cross sections helps to explain why they are larger in magnitude than the positron cross sections at those energies. This magnitude difference in the electron-positron cross section holds even at the highest energies (around a few hundred $\mathrm{eV}$ ), where one would expect them to merge. It is, in fact, at those higher energies that the scattering processes that differentiate positron from electron scattering, i.e., Ps formation and the exchange interaction, respectively, become negligible. This final observation once again points toward more progress still being needed in the future development of theoretical models for electron and, perhaps mainly, positron scattering.

\section{CONCLUSIONS}

We presented experimental positron- $\mathrm{N}_{2} \mathrm{O}$ TCSs at incident energies between 0.1 and $70 \mathrm{eV}$ and theoretical cross sections 
calculated with the SMC approach from 0.1 to $10 \mathrm{eV}$, as well as with the IAM-SCAR method at energies in the range $1-1000 \mathrm{eV}$. Our measurements extended the available range of TCS data and improved the accuracy of the existing experimental TCS thanks to the better angular discrimination of the Trento spectrometer. In addition, the present IAMSCAR calculations have included the contribution of the Ps formation channel. Our measured and computed TCSs and elastic ICS were found to be in good qualitative agreement with each other and with the earlier positron- $\mathrm{N}_{2} \mathrm{O}$ results. However, the lack of a quantitative level of accord, in particular at the lowest investigated energies, suggests that further development of the current scattering models is needed. We also presented a comparison of the present positron cross sections with a selection of the existing electron-impact results for $\mathrm{N}_{2} \mathrm{O}$ and a new electron TCS computed as a part of this study. The positron cross sections were generally found to be smaller in magnitude than the electron-impact results, although they seemed to share a qualitative energy dependence at incident energies smaller than $\sim 1 \mathrm{eV}$. This observation was interpreted as an indication for the dominant role played by the dipole interaction in the scattering process at those low energies.

\section{ACKNOWLEDGMENTS}

The experimental work was conducted under a Memorandum of Understanding between the University of Trento and the Flinders University node of the Australian Research Council (ARC) Centre of Excellence for Antimatter-Matter Studies. G.G. and F.B. would like to acknowledge the Spanish Ministerio de Economía y Productividad (Project No. FIS2009-10245) and they and M.J.B. would like to acknowledge the European Science Foundation (COST Action MP1002-Nano-IBCT) for financial support. M.H.F.B., S.d'A.S., M.T.do N.V., and M.A.P.L. would like to thank the Brazilian agency Conselho Nacional de Desenvolvimento Científico e Tecnológico (CNPq). M.H.F.B. acknowledges support from FINEP (under Project No. CT-Infra). S.d'A.S. also thanks the Fundação Araucária. M.T.do N.V., and M.A.P.L. acknowledge support from FAPESP. M.H.F.B. and S.d'A.S. would like to thank Professor Carlos M. de Carvalho at LFTC-DFis-UFPR and LCPAD-UFPR for computational support. The Brazilian authors also acknowledge computational support from CENAPAD-SP. Finally, the authors would like to thank Christof Janssen for bringing the issue of the thermal transpiration correction to their attention.
[1] P. Forster, V. Ramaswamy, P. Artaxo, T. Berntsen, R. Betts, D. W. Fahey, J. Haywood, J. Lean, D. C. Lowe, G. Myhre, J. Nganga, R. Prinn, G. Raga, M. Schulz, and R. Van Dorland, in Climate Change 2007: The Physical Science Basis. Contribution of Working Group I to the Fourth Assessment Report of the Intergovernmental Panel on Climate Change, edited by S. Solomon, D. Qin, M. Manning, Z. Chen, M. Marquis, K. B. Averyt, M. Tignor, and H. L. Miller (Cambridge University Press, Cambridge, 2007).

[2] A. Zecca, L. Chiari, A. Sarkar, and M. J. Brunger, New J. Phys. 13, 115001 (2011).

[3] L. Chiari, A. Zecca, S. Girardi, E. Trainotti, G. García, F. Blanco, R. P. McEachran, and M. J. Brunger, J. Phys. B 45, 215206 (2012).

[4] A. Zecca, L. Chiari, E. Trainotti, D. V. Fursa, I. Bray, A. Sarkar, S. Chattopadhyay, K. Ratnavelu, and M. J. Brunger, J. Phys. B 45, 015203 (2012).

[5] A. Zecca, C. Perazzolli, N. Moser, D. Sanyal, M. Chakrabarti, and M. J. Brunger, Phys. Rev. A 74, 012707 (2006).

[6] A. Zecca, L. Chiari, E. Trainotti, A. Sarkar, S. d'A. Sanchez, M. H. F. Bettega, M. T. do N. Varella, M. A. P. Lima, and M. J. Brunger, Phys. Rev. A 85, 012707 (2012).

[7] A. Zecca, L. Chiari, E. Trainotti, D. V. Fursa, I. Bray, and M. J. Brunger, Eur. Phys. J. D 64, 317 (2011).

[8] A. Zecca, L. Chiari, A. Sarkar, K. L. Nixon, and M. J. Brunger, Phys. Rev. A 80, 032702 (2009).

[9] A. Zecca, L. Chiari, E. Trainotti, and M. J. Brunger, J. Phys. B 45, 085203 (2012).

[10] D. R. Lide, CRC Handbook of Chemistry and Physics, 79th ed. (CRC Press, Boca Raton, FL, 1998).

[11] D. R. Lide, CRC Handbook of Chemistry and Physics, 76th ed. (CRC Press, Boca Raton, FL, 1995).
[12] A. Zecca, E. Trainotti, L. Chiari, G. García, F. Blanco, M. H. F. Bettega, M. T. do N. Varella, M. A. P. Lima, and M. J. Brunger, J. Phys. B 44, 195202 (2011).

[13] Ch. K. Kwan, Y.-F. Hsieh, W. E. Kauppila, S. J. Smith, T. S. Stein, M. N. Uddin, and M. S. Dababneh, Phys. Rev. Lett. 52, 1417 (1984).

[14] D. A. Przybyla, W. Addo-Asah, W. E. Kauppila, C. K. Kwan, and T. S. Stein, Phys. Rev. A 60, 359 (1999).

[15] K. L. Baluja and A. Jain, Phys. Rev. A 45, 7838 (1992).

[16] F. Arretche, K. T. Mazon, S. E. Michelin, M.-T. Lee, and M. A. P. Lima, Phys. Rev. A 77, 042708 (2008).

[17] M. Vinodkumar and M. Barot, J. Chem. Phys. 137, 074311 (2012).

[18] M. Kubo, D. Matsunaga, K. Koshio, T. Suzuki, and H. Tanaka, in Atomic Collision Research in Japan, edited by Y. Hatano et al. (Society for Atomic Collision Research, Tokyo, 1981), Vol. 7 , Progress Report 4.

[19] M. Allan and T. Skalicky, J. Phys. B 36, 3397 (2003).

[20] S. M. S. da Costa and M. H. F. Bettega, Eur. Phys. J. D 3, 67 (1998).

[21] M. H. F. Bettega, C. Winstead, and V. McKoy, Phys. Rev. A 74, 022711 (2006).

[22] K. Anzai, H. Kato, M. Hoshino, H. Tanaka, Y. Itikawa, L. Campbell, M. J. Brunger, S. J. Buckman, H. Cho, F. Blanco, G. Garcia, P. Limão-Vieira, and O. Ingólfsson, Eur. Phys. J. D 66, 36 (2012).

[23] A. Zecca, L. Chiari, A. Sarkar, S. Chattopadhyay, and M. J. Brunger, Nucl. Instrum. Methods Phys. Res. B 268, 533 (2010).

[24] A. Zecca and M. J. Brunger, in Nanoscale Interactions and Their Applications: Essays in Honor of Ian McCarthy, edited by F. Wang and M. J. Brunger (Research Signpost, Trivandrum, India, 2007), p. 21. 
[25] T. Takaishi and Y. Sensui, Trans. Faraday Soc. 59, 2503 (1963).

[26] A. Zecca, E. Trainotti, L. Chiari, M. H. F. Bettega, S. d'A Sanchez, M. T. do N. Varella, M. A. P. Lima, and M. J. Brunger, J. Chem. Phys. 136, 124305 (2012).

[27] G. A. Miller, J. Phys. Chem. 67, 1359 (1963).

[28] J. Šetina, Metrologia 36, 623 (1999).

[29] T. Ibuki and N. Sugita, J. Chem. Phys. 80, 4625 (1984).

[30] J. P. Sullivan, C. Makochekanwa, A. Jones, P. Caradonna, D. S. Slaughter, J. Machacek, R. P. McEachran, D. W. Mueller, and S. J. Buckman, J. Phys. B 44, 035201 (2011).

[31] A. Hamada and O. Sueoka, J. Phys. B 27, 5055 (1994).

[32] F. Blanco and G. García, Phys. Lett. A 295, 178 (2002).

[33] F. Blanco and G. García, Phys. Rev. A 67, 022701 (2003).

[34] F. Blanco and G. García, Phys. Lett. A 317, 458 (2003).

[35] F. Blanco and G. García, Phys. Lett. A 330, 230 (2004).

[36] L. Chiari, E. Anderson, W. Tattersall, J. R. Machacek, P. Palihawadana, C. Makochekanwa, J. P. Sullivan, G. García, F. Blanco, R. P. McEachran, M. J. Brunger, and S. J. Buckman, J. Chem. Phys. 138, 074301 (2013).

[37] M. E. Riley and D. G. Truhlar, J. Chem. Phys. 63, 2182 (1975).

[38] X. Z. Zhang, J. F. Sun, and Y. F. Liu, J. Phys. B 25, 1893 (1992).

[39] G. Staszewska, D. W. Schwenke, D. Thirumalai, and D. G. Truhlar, Phys. Rev. A 28, 2740 (1983).

[40] R. P. McEachran, J. P. Sullivan, S. J. Buckman, M. J. Brunger, M. C. Fuss, A. Muñoz, F. Blanco, R. D. White, Z. Lj. Petrović, P. Limão-Vieira, and G. García, J. Phys. B 45, 045207 (2012); 45, 189502 (2012).

[41] L. Chiari, P. Palihawadana, J. R. Machacek, C. Makochekanwa, G. García, F. Blanco, R. P. McEachran, M. J. Brunger, S. J. Buckman, and J. P. Sullivan, J. Chem. Phys. 138, 074302 (2013).

[42] A. G. Sanz, M. C. Fuss, F. Blanco, Z. Mašín, J. D. Gorfinkiel, M. J. Brunger, and G. García, Phys. Rev. A (to be published).

[43] D. D. Reid and J. M. Wadehra, Phys. Rev. A 50, 4859 (1994).

[44] D. D. Reid and J. M. Wadehra, J. Phys. B 29, L127 (1996).

[45] D. D. Reid and J. M. Wadehra, J. Phys. B 30, 2318 (1997).
[46] R. P. McEachran, D. L. Morgan, A. G. Ryman, and A. D. Stauffer, J. Phys. B 10, 663 (1977).

[47] T. M. Miller and B. Bederson, in Advances in Atomic and Molecular Physics, edited by D. R. Bates and I. Estermann (Academic Press, New York, 1977), Vol. 13, p. 1.

[48] E.-A. Reinsch and W. Meyer, Phys. Rev. A 18, 1793 (1978).

[49] M. Charlton and J. W. Humberston, Positron Physics (Cambridge University Press, Cambridge, 2001).

[50] F. Blanco and G. García, J. Phys. B 42, 145203 (2009).

[51] A. Jain, J. Phys. B 21, 905 (1988).

[52] J. S. E. Germano and M. A. P. Lima, Phys. Rev. A 47, 3976 (1993).

[53] E. P. da Silva, J. S. E. Germano, and M. A. P. Lima, Phys. Rev. A 49, R1527 (1994).

[54] E. P. da Silva, J. S. E. Germano, and M. A. P. Lima, Phys. Rev. Lett. 77, 1028 (1996).

[55] E. P. da Silva, J. S. E. Germano, J. L. S. Lino, C. R. C. de Carvalho, A. P. P. Natalense, and M. A. P. Lima, Nucl. Instrum. Methods Phys. Res. B 143, 140 (1998).

[56] C. W. Bauschlicher, J. Chem. Phys. 72, 880 (1980).

[57] E. M. de Oliveira, R. F. da Costa, S. d'A Sanchez, A. P. P. Natalense, M. H. F. Bettega, M. A. P. Lima, and M. T. do N Varella, Phys. Chem. Chem. Phys. 15, 1682 (2013).

[58] K. Watanabe, T. Nakayama, and J. Mottl, J. Quant. Spectrosc. Radiat. Transfer 2, 369 (1962).

[59] R. I. Hall, A. Chutjian, and S. Trajmar, J. Phys. B 6, L365 (1973).

[60] N. W. Winter, Chem. Phys. Lett. 33, 300 (1975).

[61] H. Nakatsuji, Chem. Phys. 75, 425 (1983).

[62] H. Kawahara, D. Suzuki, H. Kato, M. Hoshino, H. Tanaka, O. Ingólfsson, L. Campbell, and M. J. Brunger, J. Chem. Phys. 131, 114307 (2009).

[63] C. Szmytkowski, G. Karwasz, and K. Maciag, Chem. Phys. Lett. 107, 481 (1984).

[64] B. M. Bode and M. S. Gordon, J. Mol. Graphics Modell. 16, 133 (1998). 